\title{
QUOTIENTS OF EXACT CATEGORIES BY CLUSTER TILTING SUBCATEGORIES AS MODULE CATEGORIES
}

\author{
LAURENT DEMONET AND YU LIU
}

\begin{abstract}
We prove that some subquotient categories of exact categories are abelian. This generalizes a result by Koenig-Zhu in the case of (algebraic) triangulated categories. As a particular case, if an exact category $\mathcal{B}$ with enough projectives and injectives has a cluster tilting subcategory $\mathcal{M}$, then $\mathcal{B} / \mathcal{M}$ is abelian. More precisely, it is equivalent to the category of finitely presented modules over $\underline{\mathcal{M}}$.
\end{abstract}

\section{INTRODUCTION}

Recently, cluster tilting theory (see for example [6, 9, 15]) permitted to construct abelian categories from some triangulated categories. The main aim of this article is to provide a framework generalizing this observation to exact categories. By Buan-Marsh-Reiten [7, Theorem 2.2] in cluster categories, by Keller-Reiten [19, Proposition 2.1] in the 2-Calabi-Yau case and then by Koenig-Zhu [20, Theorem 3.3] in the general case, one can pass from triangulated categories to abelian categories by factoring out any cluster tilting subcategory.

The main aim of this article is to prove the analogous result for exact categories: The quotient of an exact category with enough projectives by a cluster tilting subcategory is abelian. This new result seems a priori less surprising than the one in triangulated categories because these ones are intuitively further to abelian categories. Notice that, applied to Frobenius categories, it is equivalent to the result for algebraic triangulated categories (i.e. stable categories of Frobenius categories, see [10]). However, most triangulated categories appearing in representation theory turn out to be in fact algebraic, while most exact categories are not Frobenius. In this respect, the case of exact categories can be seen as a generalization of the result concerning triangulated categories, as well as a more natural version. To support this idea, we give examples coming from singularity theory and from $n$-representation-finite algebras [16] in last section of this paper.

Let $\mathcal{B}$ be an exact category with enough projectives and injectives and $\mathcal{M}$ be a full rigid subcategory of $\mathcal{B}\left(\right.$ i.e. $\operatorname{Ext}_{\mathcal{B}}^{1}(\mathcal{M}, \mathcal{M})=0$ where $\operatorname{Ext}_{\mathcal{B}}^{1}(\mathcal{M}, \mathcal{M})$ is the essential image of the bifunctor $\left.\operatorname{Ext}_{\mathcal{B}}^{1}\right)$. We denote by $\underline{\mathcal{M}}($ resp. $\overline{\mathcal{M}})$ the full subcategory of the stable category $\underline{\mathcal{B}}$ (resp. costable category $\overline{\mathcal{B}}$ ) with the same objects as $\mathcal{M}$. Let $\mathcal{M}_{L}$ (resp. $\mathcal{M}_{R}$ ) be the subcategory of

Key words and phrases. exact category, quotient, rigid, cluster tilting, abelian category, equivalence.

The first author was supported during this research by grants P10723 and 22000723 of Japan Society for Promotion of Science. 
objects $X$ which admit short exact sequences

$$
0 \rightarrow X \stackrel{d^{0}}{\rightarrow} M^{0} \stackrel{d^{1}}{\rightarrow} M^{1} \rightarrow 0 \quad\left(\text { resp. } 0 \rightarrow M_{1} \stackrel{d_{1}}{\longrightarrow} M_{0} \stackrel{d_{0}}{\rightarrow} X \rightarrow 0\right)
$$

with $M^{0}, M^{1}, M_{0}, M_{1} \in \mathcal{M}$. In this case, $d^{0}$ (resp. $d_{0}$ ) is a left (resp. right) $\mathcal{M}$-approximation of $X$. Finally, $\bar{\Omega} \mathcal{M}$ is the class of objects $X \in \mathcal{B}$ such that there exists a short exact sequence

$$
0 \rightarrow M \rightarrow I \rightarrow X \rightarrow 0
$$

where $M \in \mathcal{M}$, and $I$ is injective.

The main result of this paper (theorems 3.2 and 3.5 ) is:

Theorem. There are equivalences of categories:

(1) between the subquotient category $\mathcal{M}_{L} /[\mathcal{M}]$ of $\mathcal{B}$ and the category of finitely presented modules $\bmod \underline{\mathcal{M}}$ if $\mathcal{M}$ contains all projective objects of $\mathcal{B}$;

(2) between the subquotient category $\mathcal{M}_{R} /[\bar{\Omega} \mathcal{M}]$ of $\mathcal{B}$ and the category of finitely presented modules $\bmod \overline{\mathcal{M}}$ if $\mathcal{M}$ contains all injective objects of $\mathcal{B}$.

This permits to get the following corollary (see Lemma 2.3):

Corollary. If $\mathcal{M}$ is contravariantly finite, then $\mathcal{M}_{L} /[\mathcal{M}]$ is abelian.

For the sake of generality, note that we have to consider categories of modules over additive categories. For more details about these, see [2, 4].

The previous results concern categories $\mathcal{M}_{L}$ and $\mathcal{M}_{R}$ which have not good properties in general. From now on, we suppose that $\mathcal{M}$ is $n$-cluster tilting for some integer $n \geq 2$ (see [14, 15]). Thus, the properties of $\mathcal{M}_{L}$ and $\mathcal{M}_{R}$ becomes much clearer: in particular,

$$
\begin{aligned}
& \mathcal{M}_{L}={ }^{\perp_{n-2}} \mathcal{M}=\left\{X \in \mathcal{B} \mid \forall i \in\{1, \ldots, n-2\}, \operatorname{Ext}_{\mathcal{B}}^{i}(X, \mathcal{M})=0\right\} \\
& \text { and } \mathcal{M}_{R}=\mathcal{M}^{\perp_{n-2}}=\left\{X \in \mathcal{B} \mid \forall i \in\{1, \ldots, n-2\}, \operatorname{Ext}_{\mathcal{B}}^{i}(\mathcal{M}, X)=0\right\} \text {, }
\end{aligned}
$$

and therefore they are exact subcategories of $\mathcal{B}$ (see Remark 3.8). In particular we get

Corollary. If $\mathcal{M}$ is 2-cluster tilting then $\mathcal{B} /[\mathcal{M}] \simeq \bmod \underline{\mathcal{M}}$ is abelian.

As a particular case of this corollary, we obtain (see Example 4.1)

Corollary. Let $S=\mathbb{C} \llbracket X, Y, Z \rrbracket$ and $G$ be a finite subgroup of $\mathrm{GL}_{3}(\mathbb{C})$ without pseudo-reflections acting on $S$. We suppose that $R=S^{G}$ is an isolated singularity. Then, denoting the category of Cohen-Macaulay modules over $R$ by $\mathrm{CM}(R)$,

$$
\frac{\mathrm{CM}(R)}{[S]} \simeq \bmod \left(\frac{S * G}{(e)}\right)^{\mathrm{op}}
$$

where $S * G$ is the skew-group algebra and the idempotent e corresponds to the trivial representation of $G$.

For example, if $G=\langle\operatorname{diag}(\zeta, \zeta, \zeta)\rangle$ where $\zeta$ is a primitive $n$-th root of the unity, then

$$
\frac{\mathrm{CM}(R)}{[S]} \simeq \bmod \mathbb{C} Q / I
$$


where

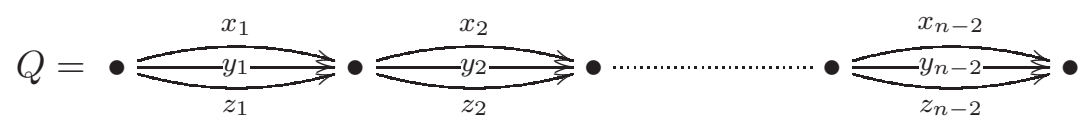

and $I$ is the ideal of $\mathbb{C} Q$ generated by the relations $x_{i} y_{i+1}=y_{i} x_{i+1}, y_{i} z_{i+1}=$ $z_{i} y_{i+1}, z_{i} x_{i+1}=x_{i} z_{i+1}$ for $1 \leq i \leq n-3$.

Moreover, if $\mathcal{B}$ has an $(n-1)$-AR translation $\tau_{n-1}$ as defined in [14, we can combine the two equivalences in the following commutative diagram of equivalences (see Theorem 3.14):

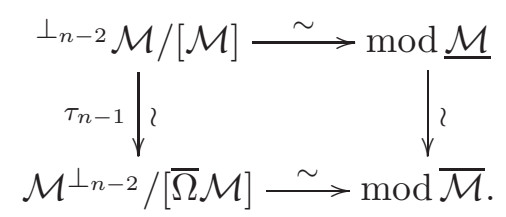

By duality, if we denote by $\bmod ^{\prime} \underline{\mathcal{M}}\left(\operatorname{resp} \cdot \bmod ^{\prime} \overline{\mathcal{M}}\right)$ the category of finitely copresented modules over $\underline{\mathcal{M}}$ (resp. $\overline{\mathcal{M}}$ ), we get the following commutative diagram:

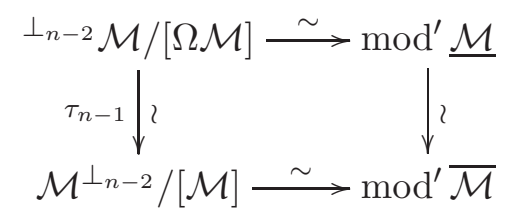

where $\Omega \mathcal{M}$ the class of objects $X \in \mathcal{B}$ such that there exists a short exact sequence

$$
0 \rightarrow X \rightarrow P \rightarrow M \rightarrow 0
$$

with $M \in \mathcal{M}$ and $P$ projective.

In Section 2, we collect basic material on exact categories. Section 3 contains main results and their proofs. In Section 4, we discuss several examples.

\section{Preliminaries}

2.1. Exact categories. In this section, we briefly review the essential properties of exact categories. For more details, we refer to [8], [18, Appendix A] and [21. Let $\mathcal{B}$ be an additive category. Let us call weak short exact sequences of $\mathcal{B}$ pairs of morphisms $(i, d)$ such that $i$ is a kernel of $d$ and $d$ a cokernel of $i$. Let $\mathcal{S}$ be a class of weak short exact sequences of $\mathcal{B}$, stable under isomorphisms, direct sums and direct summands. If a weak short exact sequence $(i, d)$ is in $\mathcal{S}$, we call it a short exact sequence, denote it by

$$
0 \rightarrow X \stackrel{i}{\rightarrow} Y \stackrel{d}{\rightarrow} Z \rightarrow 0,
$$

and we call $i$ an inflation and $d$ a deflation. The class $\mathcal{S}$ is called an exact structure on $\mathcal{B}$ (and $\mathcal{B}$ is said to be an exact category) if it satisfies the following properties, equivalent to the original axioms:

- Identity morphisms are inflations and deflations.

- The composition of two inflations (resp. deflations) is an inflation (resp. deflation). 
- If $0 \rightarrow X \stackrel{i}{\rightarrow} Y \stackrel{d}{\rightarrow} Z \rightarrow 0$ is a short exact sequence, for any morphisms $f: Z^{\prime} \rightarrow Z$ and $g: X \rightarrow X^{\prime}$, there are commutative diagrams
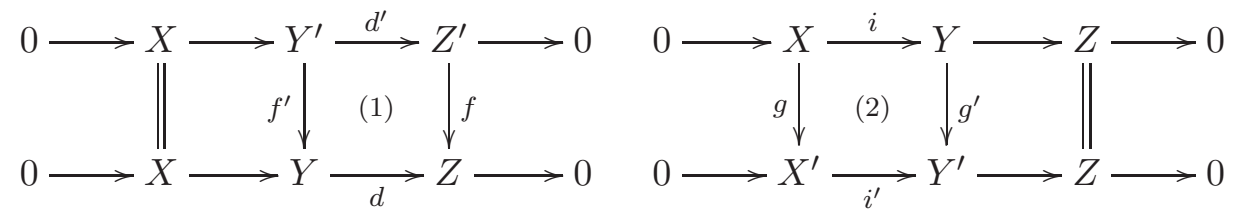

the rows of which are short exact sequences, the square (1) being a pull-back and (2) being a push-out. In this case, we easily deduce from previous points that

$$
\begin{aligned}
0 & \rightarrow Y^{\prime} \stackrel{\left(\begin{array}{l}
f^{\prime} \\
d^{\prime}
\end{array}\right)}{\longrightarrow} Y \oplus Z^{\prime} \stackrel{(-d f)}{\longrightarrow} Z \rightarrow 0 \\
\text { and } \quad 0 & \rightarrow X \stackrel{\left(\begin{array}{c}
g \\
i
\end{array}\right)}{\longrightarrow} X^{\prime} \oplus Y \stackrel{\left(-i^{\prime} g^{\prime}\right)}{\longrightarrow} Y^{\prime} \rightarrow 0
\end{aligned}
$$

are short exact sequences.

From the definition of the exact category, we get the following lemma.

Lemma 2.1. If $0 \rightarrow X \stackrel{i}{\rightarrow} Y \stackrel{d}{\rightarrow} Z \rightarrow 0$ and $0 \rightarrow Y \stackrel{f}{\rightarrow} W \rightarrow V \rightarrow 0$ are two short exact sequences, then there is a commutative diagram of short exact sequences

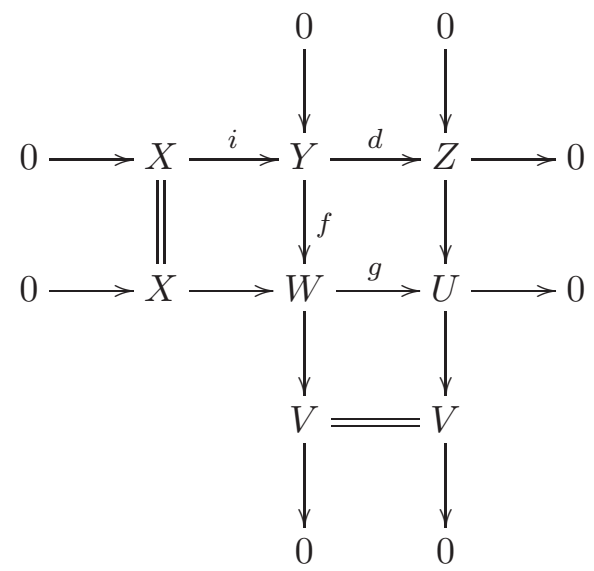

where the upper-right square is both a push-out and a pull-back.

Proof. Since $f: Y \rightarrow W$ is an inflation, there exists a push-out

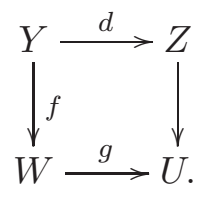

Then we get a short exact sequence $0 \rightarrow Y \rightarrow Z \oplus W \rightarrow U \rightarrow 0$, and thus the above diagram is also a pull-back. Now, if $g^{\prime}: V^{\prime} \rightarrow W$ is a morphism such that $g g^{\prime}=0$, then by the definition of a pull-back diagram, there exists 
a morphism $h: V^{\prime} \rightarrow Y$

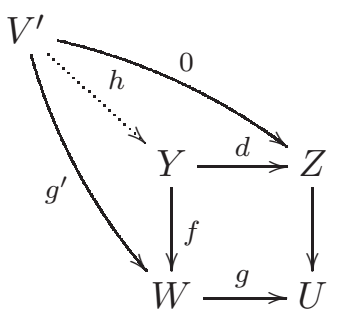

such that $g^{\prime}=f h$ and $d h=0$. Thus $h$ factors through $i$, and therefore $g^{\prime}$ factors through $f i$. Finally, as $f i$ is an inflation, $f i$ is a kernel of $g$. If $l: W \rightarrow N$ satisfies $l f i=0$, then there exists a unique morphism $k: Z \rightarrow N$ such that $l f=k d$, since $d$ is the cokernel of $i$. Then by definition of a push-out diagram, there is a unique morphism $s: U \rightarrow N$ such that $l=s g$ :

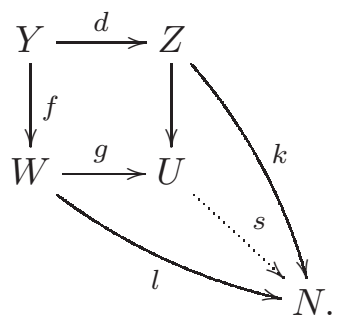

Hence $g$ is the cokernel of $f i$.

An object $P \in \mathcal{B}$ is said to be projective if for any deflation $d, \operatorname{Hom}_{\mathcal{B}}(P, d)$ is surjective. A projective cover of an object $X \in \mathcal{B}$ is a deflation $P \rightarrow X$ where $P$ is projective. An object $I \in \mathcal{B}$ is said to be injective if for any inflation $i, \operatorname{Hom}_{\mathcal{B}}(i, I)$ is surjective. An injective envelope of an object $X \in \mathcal{B}$ is an inflation $X \rightarrow I$ where $I$ is injective. The subcategory of projectives (resp. injectives) is denoted by $\mathcal{P}$ (resp. $\mathcal{I}$ ).

As for abelian categories, $\mathcal{B}$ is said to have enough injectives (resp. enough projectives) if every object admits an injective envelope (resp. a projective cover).

As in abelian category, if $\mathcal{B}$ has enough injectives (resp. projectives), we can consider injective (resp. projective) resolutions and Ext functors as rightderived functors of Hom. Thus, for all $X, Y \in \mathcal{B}, \operatorname{Ext}_{\mathcal{B}}^{1}(Y, X)$ parameterizes short exact sequences $0 \rightarrow X \rightarrow Z \rightarrow Y \rightarrow 0$ up to equivalence.

2.2. Cluster tilting subcategories. From now on, let $\mathcal{B}$ be a Hom-finite Krull-Schmidt $k$-linear exact category with enough injectives and projectives.

Recall that a subcategory $\mathcal{M}$ is called contravariantly (resp. covariantly) finite in $\mathcal{B}$ if any object $B$ of $\mathcal{B}$ admits a right (resp. left) $\mathcal{M}$-approximation, that is a morphism $f: M \rightarrow B$ (resp. $B \rightarrow M$ ), where $M \in \mathcal{M}$ such that every morphism from $\mathcal{M}$ to $B$ (resp. from $B$ to $\mathcal{M}$ ) factors through $f$.

A subcategory $\mathcal{M}$ of $\mathcal{B}$ is called n-rigid if

$$
\forall i \in\{1,2, \ldots, n-1\}, \operatorname{Ext}_{\mathcal{B}}^{i}(\mathcal{M}, \mathcal{M})=0 .
$$

$\mathcal{M}$ is called $n$-cluster tilting, if it satisfies the following conditions:

(1) $\mathcal{M}$ is contravariantly finite and covariantly finite in $\mathcal{B}$,

(2) $X \in \mathcal{M}$ if and only if $\operatorname{Ext}_{\mathcal{B}}^{i}(X, \mathcal{M})=0, \forall i \in\{1,2, \ldots, n-1\}$, 
(3) $X \in \mathcal{M}$ if and only if $\operatorname{Ext}_{\mathcal{B}}^{i}(\mathcal{M}, X)=0, \forall i \in\{1,2, \ldots, n-1\}$.

Clearly all $n$-cluster tilting subcategories are $n$-rigid. The unique 1 -cluster tilting subcategory is $\mathcal{B}$. By definition, if $\mathcal{M}$ is $n$-cluster tilting, then $\mathcal{P} \subseteq \mathcal{M}$, $\mathcal{I} \subseteq \mathcal{M}$.

2.3. The categories of modules over (co-)stable subcategories of $\mathcal{B}$. For any object $X, Y \in \mathcal{B}$ and a full subcategory $\mathcal{C}$ of $\mathcal{B}$, denote by $[\mathcal{C}](X, Y)$ the set of morphisms in $\operatorname{Hom}_{\mathcal{B}}(X, Y)$ which factor through objects of $\mathcal{C}$. If $\mathcal{P} \subseteq \mathcal{C}($ resp. $\mathcal{I} \subseteq \mathcal{C})$, the $($ co-) stable category $\underline{\mathcal{C}}$ (resp. $\overline{\mathcal{C}}$ ) of $\mathcal{C}$ is the quotient category $\mathcal{C} /[\mathcal{P}]($ resp. $\mathcal{C} /[\mathcal{I}])$, i.e. the category which has the same objects than $\mathcal{C}$ and morphisms are defined as

$$
\begin{gathered}
\underline{\operatorname{Hom}}_{\mathcal{C}}(X, Y):=\operatorname{Hom}_{\mathcal{C}}(X, Y) /[\mathcal{P}](X, Y) \\
\text { (resp. } \left.\overline{\operatorname{Hom}}_{\mathcal{C}}(X, Y):=\operatorname{Hom}_{\mathcal{C}}(X, Y) /[\mathcal{I}](X, Y)\right) .
\end{gathered}
$$

We denote the residue class of any morphism $f \in \operatorname{Hom}_{\mathcal{C}}(X, Y)$ by $\underline{f}$ (resp. $\bar{f}$ ) in $\underline{\mathcal{C}}$ (resp. $\overline{\mathcal{C}}$ ). Denote by $\operatorname{Mod} \mathcal{C}$ the category of contravariant additive functors from $\mathcal{C}$ to $\bmod k$ for any category $\mathcal{C}$. Let $\bmod \mathcal{C}$ be the full subcategory of $\operatorname{Mod} \mathcal{C}$ consisting of objects $A$ admitting an exact sequence:

$$
\operatorname{Hom}_{\mathcal{C}}\left(-, C_{1}\right) \stackrel{\beta}{\rightarrow} \operatorname{Hom}_{\mathcal{C}}\left(-, C_{0}\right) \stackrel{\alpha}{\rightarrow} A \rightarrow 0
$$

where $C_{0}, C_{1} \in \mathcal{C}$.

Remark 2.2. $\operatorname{Hom}_{\mathcal{C}}(-, C)$ is projective in $\operatorname{Mod} \mathcal{C}$ for any $C \in \mathcal{C}$.

Lemma 2.3. For any contravariantly finite subcategory $\mathcal{C}$ of $\mathcal{B}$ which contains $\mathcal{P}$, $\bmod \underline{\mathcal{C}}$ is an abelian category.

Proof. It is enough to show that $\underline{\mathcal{C}}$ has pseudokernels (see [4, §2]). Consider a morphism $f \in \operatorname{Hom}_{\mathcal{B}}\left(C_{1}, C_{0}\right)$. Let

$$
0 \rightarrow K \rightarrow P_{0} \stackrel{f^{\prime}}{\rightarrow} C_{0} \rightarrow 0
$$

be a short exact sequence with $P_{0}$ projective. Then there is a commutative diagram of exact sequences:

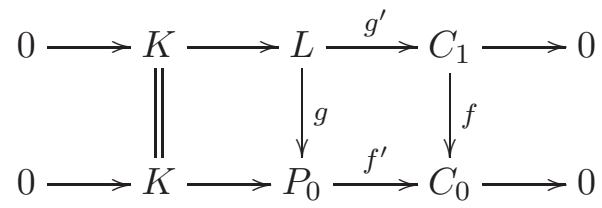

where the right square is a pull-back and a short exact sequence:

$$
0 \rightarrow L \stackrel{\left(\begin{array}{c}
-g^{\prime} \\
g
\end{array}\right)}{\longrightarrow} C_{1} \oplus P_{0} \stackrel{\left(f f^{\prime}\right)}{\longrightarrow} C_{0} \rightarrow 0 .
$$

Let $k: C_{L} \rightarrow L$ be a right $\mathcal{C}$-approximation of $L$. We claim that $g^{\prime} k$ is a pseudokernel of $\underline{f}$. If $\underline{f h}=0$ for a morphism $\underline{h} \in \underline{\operatorname{Hom}}_{\mathcal{B}}\left(C, C_{1}\right)$ with $\bar{C} \in \mathcal{C}$, then there exists an commutative diagram:

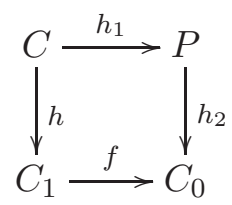


where $P$ is projective. As $f^{\prime}$ is a deflation, there exists a morphism $h^{\prime}: P \rightarrow$ $P_{0}$ such that $f^{\prime} h^{\prime}=h_{2}$. Since

$$
\left(\begin{array}{c}
h \\
-h^{\prime} h_{1}
\end{array}\right) \in \operatorname{Hom}_{\mathcal{B}}\left(C, C_{1} \oplus P_{0}\right)
$$

and

$$
\left(\begin{array}{ll}
f \quad f^{\prime}
\end{array}\right) \circ\left(\begin{array}{c}
h \\
-h^{\prime} h_{1}
\end{array}\right)=f h-f^{\prime} h^{\prime} h_{1}=f h-h_{2} h_{1}=0
$$

$\left(\begin{array}{c}h \\ -h^{\prime} h_{1}\end{array}\right)$ factors through $\left(\begin{array}{c}-g^{\prime} \\ g\end{array}\right)$. Since $k$ is a right $\mathcal{C}$-approximation of $L$, we have the following commutative diagram:

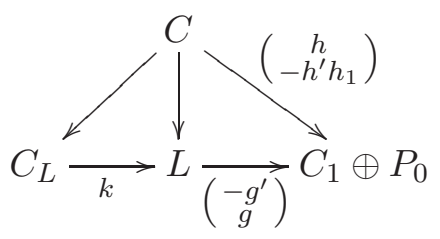

and $\underline{h}$ factors though $\underline{g^{\prime} k}$.

\section{MAIN RESUlts}

3.1. Quotient category of $\mathcal{M}_{L}$ by a rigid subcategory $\mathcal{M}$. In this subsection, we assume that $\mathcal{M}$ is a rigid subcategory of $\mathcal{B}$ which contains $\mathcal{P}$. Recall that $\mathcal{M}_{L}$ is the subcategory of objects $X$ of $\mathcal{B}$ which admit a short exact sequence $0 \rightarrow X \stackrel{d^{0}}{\rightarrow} M^{0} \stackrel{d^{1}}{\rightarrow} M^{1} \rightarrow 0$ where $M^{0}, M^{1} \in \mathcal{M}$. Now we consider the functor

$$
\begin{aligned}
H: & \mathcal{M}_{L} \rightarrow \operatorname{Mod} \underline{\mathcal{M}} \\
& \left.X \mapsto \operatorname{Ext}_{\mathcal{B}}^{1}(-, X)\right|_{\mathcal{M}}
\end{aligned}
$$

From now on, we will consider the quotient category $\mathcal{M}_{L} /[\mathcal{M}]$ and we will denote by $[f]$ the residue class in $\mathcal{M}_{L} /[\mathcal{M}]$ of any morphism $f$ of $\mathcal{M}_{L}$.

Let $\pi: \mathcal{M}_{L} \rightarrow \mathcal{M}_{L} /[\mathcal{M}]$ be the projection functor. By definition of a rigid subcategory, $H X=0$ if $X \in \mathcal{M}$. Hence, by the universal property of $\pi$, there exists a functor $F: \mathcal{M}_{L} /[\mathcal{M}] \rightarrow \operatorname{Mod} \underline{\mathcal{M}}$ such that the following diagram commutes:

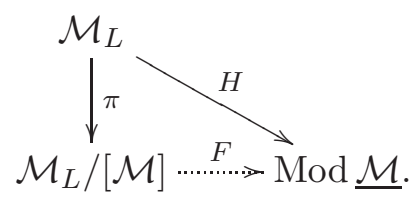

Lemma 3.1. For any short exact sequence

$$
0 \rightarrow X \stackrel{d^{0}}{\rightarrow} M^{0} \stackrel{d^{1}}{\rightarrow} M^{1} \rightarrow 0
$$

where $M^{0}, M^{1} \in \mathcal{M}$, there is an exact sequence in $\operatorname{Mod} \underline{\mathcal{M}}$

$$
\underline{\operatorname{Hom}}_{\mathcal{M}}\left(-, M^{0}\right) \rightarrow \underline{\operatorname{Hom}}_{\mathcal{M}}\left(-, M^{1}\right) \rightarrow H X \rightarrow 0 .
$$

Thus, $F X=H X \in \bmod \underline{\mathcal{M}}$. 
Proof. Applying $\operatorname{Hom}_{\mathcal{B}}(M,-)$ where $M \in \mathcal{M}$ to the short exact sequence, we get a long exact sequence:

$$
\operatorname{Hom}_{\mathcal{B}}\left(M, M^{0}\right) \stackrel{\operatorname{Hom}_{\mathcal{B}}\left(M, d^{1}\right)}{\longrightarrow} \operatorname{Hom}_{\mathcal{B}}\left(M, M^{1}\right) \stackrel{\delta}{\rightarrow} \operatorname{Ext}_{\mathcal{B}}^{1}(M, X) \rightarrow 0 .
$$

This means that the following sequence in $\bmod \mathcal{M}$ is exact:

$$
\operatorname{Hom}_{\mathcal{M}}\left(-, M^{0}\right) \rightarrow \operatorname{Hom}_{\mathcal{M}}\left(-, M^{1}\right) \rightarrow H X \rightarrow 0 .
$$

As $d^{1}$ is a deflation, any morphism $g \in[\mathcal{P}]\left(M, M^{1}\right)$ factors through $d^{1}$. Hence $\delta(g)=0$. Thus $\delta$ induces a morphism $\underline{\delta}: \underline{\operatorname{Hom}}_{\mathcal{B}}\left(M, M^{1}\right) \rightarrow$ $H X(M)=\operatorname{Ext}_{\mathcal{B}}^{1}(M, X): \underline{\delta}(f)=\delta(f)$. For any $M \in \overline{\mathcal{M}}, \underline{\delta}$ is surjective. Moreover, since $\delta \circ \operatorname{Hom}_{\mathcal{B}}\left(\bar{M}, d^{1}\right)=0$, we get $\underline{\delta} \circ \underline{\operatorname{Hom}}_{\mathcal{B}}\left(M, d^{1}\right)=0$. If $\underline{\delta}(\underline{g})=0$ for a morphism $g \in \operatorname{Hom}_{\mathcal{B}}\left(M, M^{1}\right)$, then by definition $\delta(g)=0$, there exists a morphism $h \in \operatorname{Hom}_{\mathcal{B}}\left(M, M^{0}\right)$ such that $g=f h$. Hence $\underline{g}=\underline{f h}$. Thus we get an exact sequence in $\bmod \underline{\mathcal{M}}$

$$
\underline{\operatorname{Hom}}_{\mathcal{M}}\left(-, M^{0}\right) \rightarrow \underline{\operatorname{Hom}}_{\mathcal{M}}\left(-, M^{1}\right) \rightarrow H X \rightarrow 0 .
$$

The last argument can be understood as the right exactness of the functor $\underline{\mathcal{M}} \otimes_{\mathcal{M}}-: \bmod \mathcal{M} \rightarrow \bmod \underline{\mathcal{M}}$.

Theorem 3.2. The functor $F: \mathcal{M}_{L} /[\mathcal{M}] \rightarrow \bmod \underline{\mathcal{M}}$ is an equivalence of categories.

Proof. - Let us prove that $F$ is full:

Let $X, Y \in \mathcal{M}_{L}$ and $\alpha \in \operatorname{Hom}_{\bmod } \underline{\mathcal{M}}(F X, F Y)$. Consider the following short exact sequences:

$$
\begin{gathered}
0 \rightarrow X \stackrel{d^{0}}{\rightarrow} M^{0} \stackrel{d^{1}}{\rightarrow} M^{1} \rightarrow 0 \\
0 \rightarrow Y \stackrel{e^{0}}{\rightarrow} N^{0} \stackrel{e^{1}}{\rightarrow} N^{1} \rightarrow 0
\end{gathered}
$$

where $M^{0}, M^{1}, N^{0}, N^{1} \in \mathcal{M}$. By Lemma 3.1. and because $\operatorname{Hom}_{\mathcal{M}}\left(-, M^{1}\right)$ is projective in $\operatorname{Mod} \underline{\mathcal{M}}$, we get the following diagram with exact rows in $\bmod \underline{\mathcal{M}}$ :

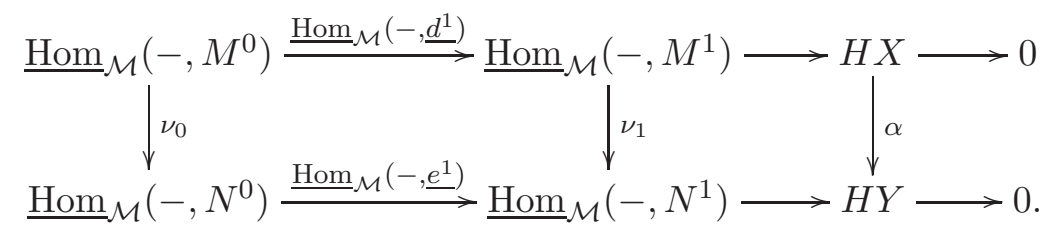

By Yoneda's Lemma, for $i=0,1$, there is a morphism $f^{i} \in \underline{\operatorname{Hom}}_{\mathcal{M}}\left(M^{i}, N^{i}\right)$ such that $\nu_{i}=\underline{\operatorname{Hom}}_{\mathcal{M}}\left(-, f^{i}\right)$ and $e^{1} f^{0}=f^{1} d^{1}$. Then there exists a projective $P \in \mathcal{M}, a \in \operatorname{Hom}_{\mathcal{M}}\left(M^{0}, \bar{P}\right)$ and $\left.\overline{b \in \operatorname{Hom}_{\mathcal{M}}(P}, N^{1}\right)$ such that $f^{1} d^{1}-e^{1} f^{0}=$ $b a$. Thus, as $e^{1}$ is a deflation, there exists $c \in \operatorname{Hom}_{\mathcal{M}}\left(P, N^{0}\right)$ such that the following diagram commutes:

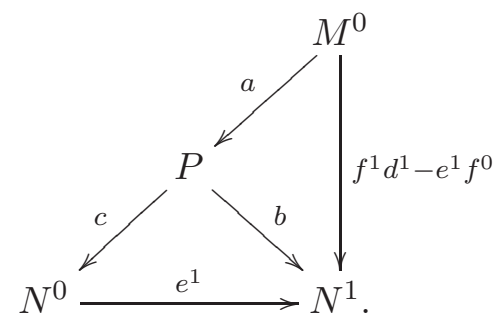


Let $g^{0}=f^{0}+c a$, then $g^{0}=f^{0}$ and $e^{1} g^{0}=f^{1} d^{1}$. Therefore, there exists $f: X \rightarrow Y$ such that $e^{0} \bar{f}=g^{0} \overline{d^{0}}$, and $\alpha=F([f])$.

- Let us prove that $F$ is faithful:

Let $f: X \rightarrow Y$ be a morphism in $\mathcal{M}_{L}$. Since $d^{0}$ is a left $\mathcal{M}$-approximation, there exists a commutative diagram of short exact sequences

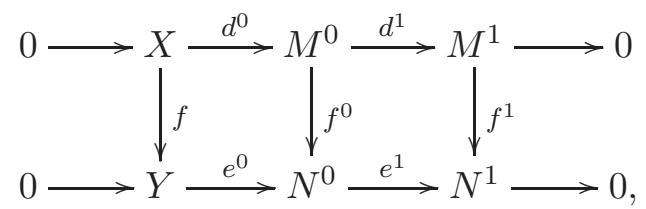

and a commutative diagram in $\bmod \underline{\mathcal{M}}$

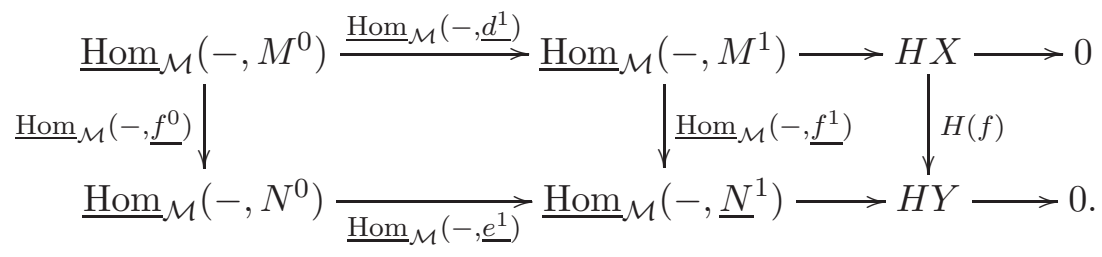

Assume that $F(\underline{f})=0$. Then $H(f)=0$ and thus, there exists a morphism $\beta$ : $\underline{\operatorname{Hom}}_{\mathcal{M}}\left(-, M^{1}\right) \rightarrow \underline{\operatorname{Hom}}_{\mathcal{M}}\left(-, N^{0}\right)$ such that $\underline{\operatorname{Hom}}_{\mathcal{M}}\left(-, \underline{f}^{1}\right)=\underline{\operatorname{Hom}}_{\mathcal{M}}\left(-, \underline{e^{1}}\right) \circ$ $\beta$. By Yoneda's Lemma, there exists a morphism $g: \bar{M}^{1} \rightarrow N^{0}$ such that $\beta=\underline{\operatorname{Hom}}_{\mathcal{M}}(-, \underline{g})$ and $\underline{f^{1}}=\underline{e^{1} g}$. Then, as before, we can complete the following commutative diagram

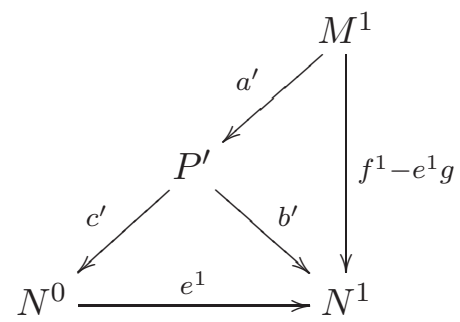

where $P^{\prime}$ is projective. Hence $e^{1}\left(c^{\prime} a^{\prime}+g\right) d^{1}=f^{1} d^{1}=e^{1} f^{0}$ and there exists a morphism $g^{\prime}: M^{0} \rightarrow Y$ such that $e^{0} g^{\prime}=f^{0}-\left(c^{\prime} a^{\prime}+g\right) d^{1}$ and $e^{0}\left(f-g^{\prime} d^{0}\right)=0$. Since $e^{0}$ is monic, $f=g^{\prime} d^{0}$, thus $[f]=0$ in $\mathcal{M}_{L} /[\mathcal{M}]$.

- Let us prove that $F$ is dense:

For any object $C \in \bmod \underline{\mathcal{M}}$, there is an exact sequence in $\bmod \underline{\mathcal{M}}$ :

$$
\underline{\operatorname{Hom}}_{\mathcal{M}}\left(-, M_{1}\right) \stackrel{\beta}{\rightarrow} \underline{\operatorname{Hom}}_{\mathcal{M}}\left(-, M_{0}\right) \rightarrow C \rightarrow 0
$$

where $M_{0}, M_{1} \in \mathcal{M}$. Since

$$
\operatorname{Hom}_{\bmod } \underline{\mathcal{M}}\left(\underline{\operatorname{Hom}}_{\mathcal{M}}\left(-, M_{1}\right), \underline{\operatorname{Hom}}_{\mathcal{M}}\left(-, M_{0}\right)\right) \simeq \underline{\operatorname{Hom}}_{\mathcal{M}}\left(M_{1}, M_{0}\right)
$$

by Yoneda's Lemma, there exists $f: M_{1} \rightarrow M_{0}$ such that $\beta=\underline{\operatorname{Hom}}_{\mathcal{M}}(-, \underline{f})$. Let

$$
0 \rightarrow K \rightarrow P_{0} \stackrel{f^{\prime}}{\rightarrow} M_{0} \rightarrow 0
$$

be a short exact sequence with $P_{0}$ projective. From the proof of Lemma 2.3, there exists a short exact sequence

$$
0 \rightarrow L \rightarrow M_{1} \oplus P_{0} \stackrel{\left(f f^{\prime}\right)}{\longrightarrow} M_{0} \rightarrow 0 .
$$


Therefore $L \in \mathcal{M}_{L}$ and there is an exact sequence:

$$
\underline{\operatorname{Hom}}_{\mathcal{M}}\left(-, M_{1}\right) \stackrel{\beta}{\rightarrow} \underline{\operatorname{Hom}}_{\mathcal{M}}\left(-, M_{0}\right) \rightarrow F L \rightarrow 0 .
$$

Since both $F L$ and $C$ are cokernels of $\beta, C \simeq F L$.

By this theorem and Lemma 2.3, we get the following corollary:

Corollary 3.3. If $\mathcal{M}$ is rigid and contravariantly finite, then $\mathcal{M}_{L} /[\mathcal{M}]$ is abelian.

3.2. Quotient category of $\mathcal{M}_{R}$ by $\bar{\Omega} \mathcal{M}$. In this subsection we assume that $\mathcal{M}$ is a rigid subcategory of $\mathcal{B}$ which contains $\mathcal{I}$. Recall that $\mathcal{M}_{R}$ is the subcategory of objects $Y$ which admit a short exact sequence

$$
0 \rightarrow N_{1} \stackrel{d_{1}^{\prime}}{\longrightarrow} N_{0} \stackrel{d_{0}^{\prime}}{\longrightarrow} Y \rightarrow 0
$$

where $N_{0}, N_{1} \in \mathcal{M}$ and $\bar{\Omega} \mathcal{M}$ the full subcategory of objects $X \in \mathcal{B}$ such that there exists a short exact sequence $0 \rightarrow M \rightarrow I \rightarrow X \rightarrow 0$ where $M \in \mathcal{M}, I \in \mathcal{I}$.

We denote

$$
\begin{aligned}
K: \mathcal{M}_{R} & \rightarrow \operatorname{Mod} \overline{\mathcal{M}} \\
X & \left.\mapsto \overline{\operatorname{Hom}}_{\mathcal{B}}(-, X)\right|_{\overline{\mathcal{M}}}
\end{aligned}
$$

Let $\pi^{\prime}: \mathcal{M}_{R} \rightarrow \mathcal{M}_{R} /[\bar{\Omega} \mathcal{M}]$ be the projection functor. By the universal property of $\pi^{\prime}$, there is a functor $G: \mathcal{M}_{R} /[\bar{\Omega} \mathcal{M}] \rightarrow \operatorname{Mod} \overline{\mathcal{M}}$ which makes the following diagram commute:

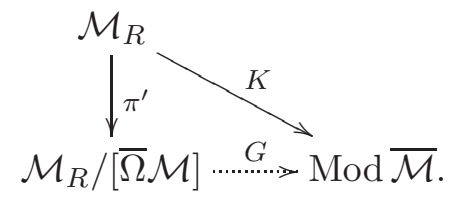

Lemma 3.4. For every $X \in \mathcal{M}_{R}, G(X)=K(X) \in \bmod \overline{\mathcal{M}}$. More precisely, for every short exact sequence

$$
0 \rightarrow M_{1} \stackrel{d_{1}}{\rightarrow} M_{0} \stackrel{d_{0}}{\rightarrow} X \rightarrow 0
$$

where $M_{1}, M_{0} \in \mathcal{M}$, there is an exact sequence

$$
K\left(M_{1}\right) \stackrel{K\left(d_{1}\right)}{\longrightarrow} K\left(M_{0}\right) \stackrel{K\left(d_{0}\right)}{\longrightarrow} K(X) \rightarrow 0 .
$$

Proof. For any nonzero object $M \in \mathcal{M}$, we have an exact sequence

$$
0 \rightarrow \operatorname{Hom}_{\mathcal{B}}\left(M, M_{1}\right) \rightarrow \operatorname{Hom}_{\mathcal{B}}\left(M, M_{0}\right) \stackrel{\operatorname{Hom}_{\mathcal{B}}\left(M, d_{0}\right)}{\longrightarrow} \operatorname{Hom}_{\mathcal{B}}(M, X) \rightarrow 0
$$

by applying $\operatorname{Hom}_{\mathcal{B}}(M,-)$. Hence $d_{0}$ is a right $\mathcal{M}$-approximation and $K\left(d_{0}\right)$ : $K\left(M_{0}\right) \rightarrow K(X)$ is surjective. If a morphism $h \in \operatorname{Hom}_{\mathcal{B}}\left(M, M_{0}\right)$ satisfies $\overline{d_{0} h}=0$ in $\overline{\mathcal{B}}$, then there exists an object $I \in \mathcal{I}$ and a commutative diagram

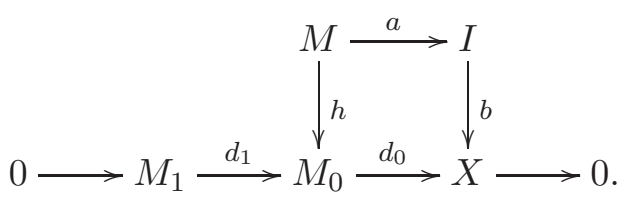


Since $I \in \mathcal{M}$, there is a morphism $c: I \rightarrow M_{0}$ such that $b=d_{0} c$. Hence $d_{0} c a=b a=d_{0} h$, and thus $d_{0}(h-c a)=0$. Therefore, $h-c a$ factors through $d_{1}$. Finally $\bar{h}$ factors through $\overline{d_{1}}$.

Theorem 3.5. The functor $G: \mathcal{M}_{R} /[\bar{\Omega} \mathcal{M}] \rightarrow \bmod \overline{\mathcal{M}}$ is an equivalence of categories.

Remark 3.6. In contrast to Corollary 3.3, we cannot conclude that $\mathcal{M}_{R} /[\bar{\Omega} \mathcal{M}]$ is abelian. Indeed, it is unlikely that a result analogous to Lemma 2.3 is true for $\bmod \overline{\mathcal{M}}$.

Proof. Let $X, Y \in M_{R}$. By definition, there exist short exact sequences

$$
\begin{gathered}
0 \rightarrow M_{1} \stackrel{d_{1}}{\rightarrow} M_{0} \stackrel{d_{0}}{\rightarrow} X \rightarrow 0 \\
0 \rightarrow N_{1} \stackrel{d_{1}^{\prime}}{\rightarrow} N_{0} \stackrel{d_{0}^{\prime}}{\longrightarrow} Y \rightarrow 0 .
\end{gathered}
$$

- Let us show that $K$ (and therefore $G$ ) is full:

Let $\alpha: K(X) \rightarrow K(Y)$ be a morphism in $\bmod \overline{\mathcal{M}}$. By Lemma 3.4, and because $K\left(M_{0}\right)$ is projective, we can form the following commutative diagram

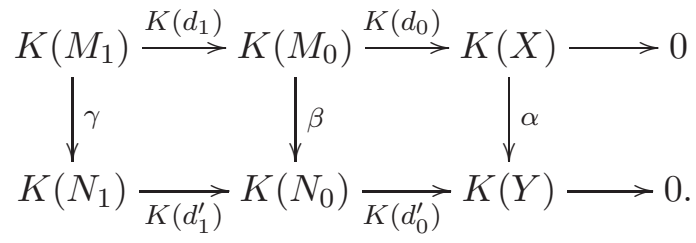

By Yoneda's Lemma, there exist $g: M_{0} \rightarrow N_{0}$ and $h: M_{1} \rightarrow N_{1}$ such that $\beta=K(g)$ and $\gamma=K(h)$. Hence $\overline{g d_{1}}=\overline{d_{1}^{\prime} h}$, and therefore $g d_{1}-d_{1}^{\prime} h$ factors through some object $I \in \mathcal{I}$. As $d_{1}$ is an inflation, we can complete the following commutative diagram:

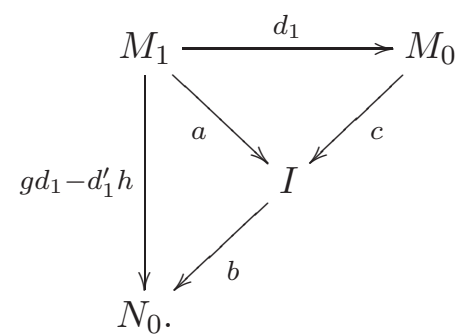

Thus, putting $g^{\prime}=g-b c$, we get $g^{\prime} d_{1}=d_{1}^{\prime} h$. Hence there is a morphism $f: X \rightarrow Y$ such that the diagram

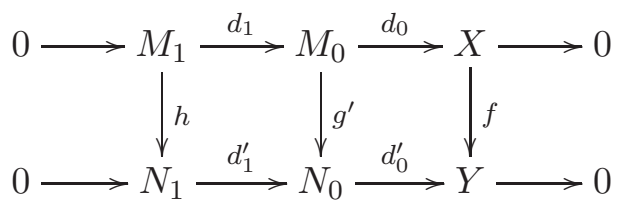

commutes. Finally, $\alpha=K(f)$.

- Let us show that $G$ is faithful:

Let $M_{0} \stackrel{i}{\rightarrow} I_{M}$ be an injective envelope of $M_{0}$. Let $f: X \rightarrow Y$ be a morphism 
of $\mathcal{M}_{R}$. If $G([f])=0$ then $K(f)=0$. Hence, $f d_{0}$ factors through some object in $\mathcal{I}$. Therefore, we have a commutative diagram

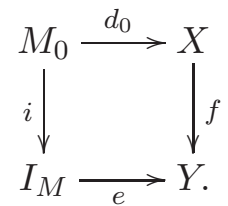

Then, if $N$ is a push-out of $i$ and $d_{0}$, we can form the commutative diagram

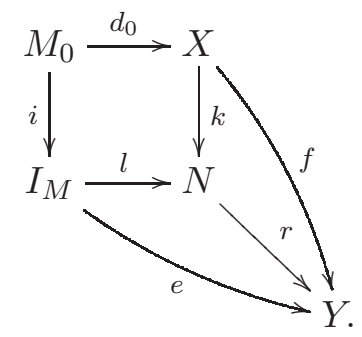

By Lemma 2.1, there is a short exact sequence

$$
0 \rightarrow M_{1} \rightarrow I_{M} \rightarrow N \rightarrow 0 .
$$

Hence $N \in \bar{\Omega} \mathcal{M}$ and then $[f]=0$ in $\mathcal{M}_{R} /[\bar{\Omega} \mathcal{M}]$.

- Let us show that $G$ is dense:

For any object $C \in \bmod \overline{\mathcal{M}}$, there is an exact sequence

$$
K\left(M_{1}\right) \stackrel{K(d)}{\longrightarrow} K\left(M_{0}\right) \rightarrow C \rightarrow 0 .
$$

First, let us form a push-out diagram

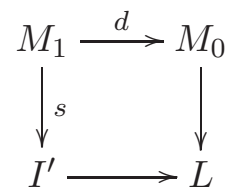

where $s$ is an injective envelope of $M_{1}$. Then

$$
0 \rightarrow M_{1} \rightarrow I^{\prime} \oplus M_{0} \rightarrow L \rightarrow 0
$$

is a short exact sequence. By Lemma 3.4, we get an exact sequence

$$
K\left(M_{1}\right) \rightarrow K\left(M_{0}\right) \rightarrow K(L) \rightarrow 0 .
$$

Hence $C \simeq K(L)=G(L)$.

If we denote $\overline{\mathcal{M}}^{\perp}=\left\{X \in \mathcal{M}_{R} \mid \overline{\operatorname{Hom}}_{\mathcal{B}}(\mathcal{M}, X)=0\right\}$, we get the following corollary:

Corollary 3.7. $\bar{\Omega} \mathcal{M}=\overline{\mathcal{M}}^{\perp}$.

Proof. By definition, $\bar{\Omega} \mathcal{M} \subseteq \overline{\mathcal{M}}^{\perp}$. If $X \in \overline{\mathcal{M}}^{\perp}$, then $G(X)=K(X)=0$. Since $G$ is faithful, $X \in \bar{\Omega} \mathcal{M}$. 
3.3. Case of $n$-cluster tilting subcategories and AR translation. In this subsection, we assume that $n>1$ and $\mathcal{M}$ is an $n$-cluster tilting subcategory.

Define

$$
\begin{aligned}
\perp_{n-2} \mathcal{M} & =\left\{X \in \mathcal{B} \mid \forall i \in\{1, \ldots, n-2\}, \operatorname{Ext}_{\mathcal{B}}^{i}(X, \mathcal{M})=0\right\} \\
\text { and } \quad \mathcal{M}^{\perp_{n-2}} & =\left\{X \in \mathcal{B} \mid \forall i \in\{1, \ldots, n-2\}, \operatorname{Ext}_{\mathcal{B}}^{i}(\mathcal{M}, X)=0\right\}
\end{aligned}
$$

Remark 3.8. The categories ${ }^{\perp_{n-2}} \mathcal{M}$ and $\mathcal{M}^{\perp_{n-2}}$ are extension closed and thus exact subcategories of $\mathcal{B}$.

Proposition 3.9. The following equalities hold:

$$
{ }^{\perp_{n-2}} \mathcal{M}=\mathcal{M}_{L} \quad \text { and } \quad \mathcal{M}^{\perp_{n-2}}=\mathcal{M}_{R} .
$$

Proof. We only prove the first equality. Let $X \in \perp_{n-2} \mathcal{M}$ and $0 \rightarrow X \stackrel{d_{0}}{\rightarrow}$ $I_{0} \rightarrow C \rightarrow 0$ be a short exact sequence with $I_{0} \in \mathcal{I}$. By definition of an exact category, we get the following commutative diagram

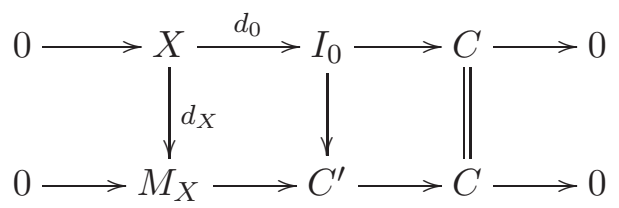

where $d_{X}$ is a left $\mathcal{M}$-approximation and the first square is a push-out. Moreover, we have a short exact sequence

$$
0 \rightarrow X \stackrel{\left(\begin{array}{l}
d_{X} \\
d_{0}
\end{array}\right)}{\longrightarrow} M_{X} \oplus I_{0} \rightarrow C^{\prime} \rightarrow 0 .
$$

If $M \in \mathcal{M}$, we have an exact sequence

$$
\operatorname{Hom}_{\mathcal{B}}\left(M_{X} \oplus I_{0}, M\right) \rightarrow \operatorname{Hom}_{\mathcal{B}}(X, M) \rightarrow \operatorname{Ext}_{\mathcal{B}}^{1}\left(C^{\prime}, M\right) \rightarrow 0
$$

since $\operatorname{Ext}_{\mathcal{B}}^{1}\left(M_{X} \oplus I_{0}, M\right)=0$. Moreover, $\operatorname{Hom}_{\mathcal{B}}\left(d_{X}, M\right)$ is surjective, since $d_{X}$ is a left $\mathcal{M}$-approximation. Hence $\operatorname{Ext}_{\mathcal{B}}^{1}\left(C^{\prime}, M\right)=0$.

Moreover, applying $\operatorname{Hom}_{\mathcal{B}}(-, M)$ where $M \in \mathcal{M}$ to the former short exact sequence, we get a long exact sequence

$$
\begin{aligned}
\cdots & \rightarrow \operatorname{Ext}_{\mathcal{B}}^{i}\left(M_{X} \oplus I_{0}, M\right) \rightarrow \operatorname{Ext}_{\mathcal{B}}^{i}(X, M) \rightarrow \operatorname{Ext}_{\mathcal{B}}^{i+1}\left(C^{\prime}, M\right) \\
& \rightarrow \operatorname{Ext}_{B}^{i+1}\left(M_{X} \oplus I_{0}, M\right) \rightarrow \cdots
\end{aligned}
$$

for $\forall i \in\{1,2, \ldots, n-2\}$. Since $\operatorname{Ext}_{\mathcal{B}}^{i}\left(M_{X} \oplus I_{0}, M\right)=\operatorname{Ext}_{\mathcal{B}}^{i+1}\left(M_{X} \oplus I_{0}, M\right)=$ 0 , we get $\operatorname{Ext}_{\mathcal{B}}^{i}(X, M) \simeq \operatorname{Ext}_{B}^{i+1}\left(C^{\prime}, M\right)$.

Thus, as $X \in \perp_{n-2} \mathcal{M}, \operatorname{Ext}_{\mathcal{B}}^{i}\left(C^{\prime}, M\right)=0$ if $1<i<n-1$. As $\mathcal{M}$ is $n$-cluster tilting, $C^{\prime} \in \mathcal{M}$. Finally, ${ }^{\perp_{n-2}} \mathcal{M} \subseteq \mathcal{M}_{L}$.

Suppose that $X \in \mathcal{M}_{L}$. Then it admits a short exact sequence

$$
0 \rightarrow X \rightarrow M^{0} \rightarrow M^{1} \rightarrow 0
$$

where $M^{0}, M^{1} \in \mathcal{M}$. Applying $\operatorname{Hom}_{\mathcal{B}}(-, M)$ where $M \in \mathcal{M}$, we get a long exact sequence

$$
0=\operatorname{Ext}_{\mathcal{B}}^{i}\left(M^{0}, M\right) \rightarrow \operatorname{Ext}_{\mathcal{B}}^{i}(X, M) \rightarrow \operatorname{Ext}_{\mathcal{B}}^{i+1}\left(M^{1}, M\right)=0
$$

for $\forall i \in\{1,2, \ldots, n-2\}$. Therefore, $X \in{ }^{\perp_{n-2}} \mathcal{M}$. 
For any object $M \in \mathcal{M}$, we fix a short exact sequence $0 \rightarrow M \rightarrow I_{M} \rightarrow$ $N \rightarrow 0$ with $I_{M} \in \mathcal{I}$ and denote $N$ by $\bar{\Omega} M$. Notice that $\bar{\Omega} M$ does not depend on $I_{M}$ as an object of $\overline{\bar{\Omega} \mathcal{M}}$. Moreover, if $f: M \rightarrow M^{\prime}$ is a morphism in $\mathcal{M}$ then we can form a commutative diagram:

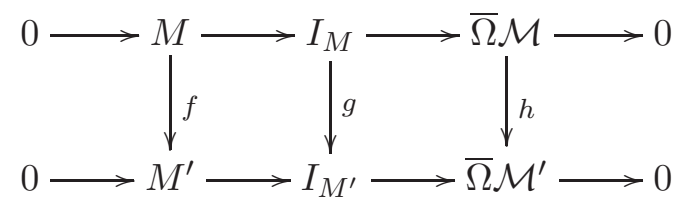

where $\overline{\Omega f}:=\bar{h}$ is uniquely determined by the morphism $\bar{f}$ in $\overline{\mathcal{M}}$. Then we get

Proposition 3.10. With the previous notation, $\bar{\Omega}: \overline{\mathcal{M}} \rightarrow \overline{\bar{\Omega} \mathcal{M}}$ is an equivalence of categories.

Now, we assume that $\mathcal{B}$ has an AR translation $\tau: \underline{\mathcal{B}} \rightarrow \overline{\mathcal{B}}$ with reciprocal $\tau^{-}$. Following [14], we define $(n-1)$-AR translations

$$
\tau_{n-1}: \underline{\perp_{n-2} \mathcal{P}} \rightarrow \overline{\mathcal{I}^{\perp_{n-2}}} \text { and } \tau_{n-1}^{-}: \overline{\mathcal{I}^{\perp_{n-2}}} \rightarrow \underline{\perp_{n-2} \mathcal{P}}
$$

by $\tau_{n-1}=\tau \Omega^{n-2}$ and $\tau_{n-1}^{-}=\tau \bar{\Omega}^{n-2}$ (where $\Omega$ is the syzygy functor). In fact, the only property we need for these functors is that, if $X \in{ }^{\perp_{n-2}} \mathcal{P}$ and $Y \in \mathcal{I}^{\perp_{n-2}}$, the following functorial isomorphisms hold:

(1) $\operatorname{Ext}_{\mathcal{B}}^{n-1}(X, Y) \simeq \mathrm{D} \overline{\operatorname{Hom}}_{\mathcal{B}}\left(Y, \tau_{n-1} X\right) \simeq \mathrm{D} \underline{\operatorname{Hom}}_{\mathcal{B}}\left(\tau_{n-1}^{-} Y, X\right)$,

(2) $\forall i \in\{1,2, \ldots, n-2\}$,

$$
\operatorname{Ext}_{\mathcal{B}}^{n-1-i}(X, Y) \simeq \operatorname{DExt}_{\mathcal{B}}^{i}\left(Y, \tau_{n-1} X\right) \simeq \operatorname{DExt}_{\mathcal{B}}^{i}\left(\tau_{n-1}^{-} Y, X\right)
$$

where $D=\operatorname{Hom}_{k}(-, k)$. This is a weak version of [14, Theorem 1.5].

From this, we deduce easily that $\tau_{n-1}$ induces an equivalence from $\underline{\perp_{n-2} \mathcal{M}}$ to $\overline{\mathcal{M}^{\perp_{n-2}}}$ the inverse of which is $\tau_{n-1}^{-1}=\tau_{n-1}^{-}$.

Remark that

$$
\begin{aligned}
& X \in \mathcal{M} \Leftrightarrow \operatorname{Ext}_{\mathcal{B}}^{i}(X, \mathcal{M})=0, \forall i \in\{1,2, \ldots, n-1\} \\
& \Leftrightarrow\left\{\begin{array}{l}
\overline{\operatorname{Hom}}_{\mathcal{B}}\left(\mathcal{M}, \tau_{n-1} X\right)=0 \\
\operatorname{Ext}_{\mathcal{B}}^{i}\left(\mathcal{M}, \tau_{n-1} X\right)=0 \text { for all } i \in\{1,2, \ldots, n-2\}
\end{array}\right. \\
& \Leftrightarrow \tau_{n-1} X \in \mathcal{M}^{\perp_{n-2}} \cap \overline{\mathcal{M}}^{\perp} \text {. }
\end{aligned}
$$

Moreover, as $\overline{\mathcal{M}}^{\perp}=\bar{\Omega} \mathcal{M} \subseteq \mathcal{M}^{\perp_{n-2}}, X \in \mathcal{M} \Leftrightarrow \tau_{n-1} X \in \bar{\Omega} \mathcal{M}$.

Now $X \in \mathcal{P}$ implies that $\operatorname{Ext}_{\mathcal{B}}^{n-1}(X, \mathcal{B})=0$, then $\overline{\operatorname{Hom}}_{\mathcal{B}}\left(\mathcal{B}, \tau_{n-1} X\right)=0$, which means $\tau_{n-1} X \in \mathcal{I}$. Dually $X \in \mathcal{I}$ implies that $\tau_{n-1}^{-1} X \in \mathcal{P}$. Hence $X \in \mathcal{P} \Leftrightarrow \tau_{n-1} X \in \mathcal{I}$. We get the following proposition

Proposition 3.11. The functor $\tau_{n-1}$ induces an equivalence from $\underline{\mathcal{M}}$ to $\overline{\bar{\Omega} \mathcal{M}}$ and an equivalence from ${ }^{\perp_{n-2}} \mathcal{M} /[\mathcal{M}]$ to $\mathcal{M}^{\perp_{n-2}} /[\bar{\Omega} \mathcal{M}]$.

Denote by $\bar{\Omega}^{-1}$ the inverse of $\bar{\Omega}: \overline{\mathcal{M}} \rightarrow \overline{\bar{\Omega} \mathcal{M}}$. Then we have

Corollary 3.12. The compositions $\tau_{n-1}^{-1} \circ \bar{\Omega}$ and $\bar{\Omega}^{-1} \circ \tau_{n-1}$ induce mutually inverse equivalences between $\overline{\mathcal{M}}$ and $\underline{\mathcal{M}}$.

According to this corollary, we can define reciprocal equivalences: 
QUOTIENTS OF EXACT CATEGORIES BY CLUSTER TILTING SUBCATEGORIES 15

(1) $\mu: \operatorname{Mod} \underline{\mathcal{M}} \rightarrow \operatorname{Mod} \overline{\mathcal{M}}, \mu(C)=C \circ \tau_{n-1}^{-1} \circ \bar{\Omega}$,

(2) $\mu^{-1}: \operatorname{Mod} \overline{\mathcal{M}} \rightarrow \operatorname{Mod} \underline{\mathcal{M}}, \mu^{-1}\left(C^{\prime}\right)=C^{\prime} \circ \bar{\Omega}^{-1} \circ \tau_{n-1}$.

Thus we get:

Proposition 3.13. The functors $\mu$ and $\mu^{-1}$ induce mutually inverse equivalences between $\bmod \underline{\mathcal{M}}$ and $\bmod \overline{\mathcal{M}}$.

Proof. We just check that $\mu$ sends an object in $\bmod \underline{\mathcal{M}}$ to an object in $\bmod \overline{\mathcal{M}}$. For any object $C \in \bmod \underline{\mathcal{M}}$, there exists an exact sequence:

$$
\underline{\operatorname{Hom}}_{\mathcal{M}}\left(-, M_{1}\right) \stackrel{\operatorname{Hom}_{\mathcal{M}}(-, f)}{\longrightarrow} \underline{\operatorname{Hom}}_{\mathcal{M}}\left(-, M_{0}\right) \rightarrow C \rightarrow 0
$$

where $M_{1}, M_{0} \in \mathcal{M}$. Hence we get the following commutative diagram:

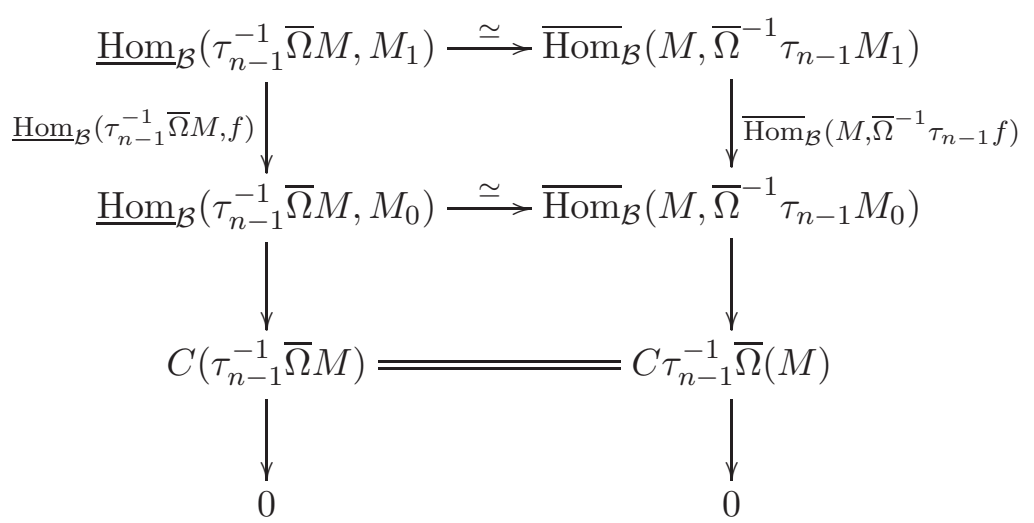

where each column is exact and $M \in \mathcal{M}$. Thus $C \tau_{n-1}^{-1} \bar{\Omega} \in \bmod \overline{\mathcal{M}}$.

Theorem 3.14. If $\mathcal{B}$ has an $(n-1)-A R$ translation $\tau_{n-1}$, then we have a diagram which is commutative up to the equivalence

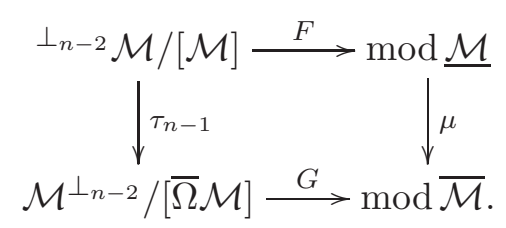

Proof. Let $M \in \mathcal{M}$ and $X \in \perp_{n-2} \mathcal{M}$. Then, applying $\operatorname{Hom}_{\mathcal{B}}(X,-)$ to $0 \rightarrow M \rightarrow I_{M} \stackrel{\alpha}{\rightarrow} \bar{\Omega} M \rightarrow 0$, we get an exact sequence:

$$
\operatorname{Hom}_{\mathcal{B}}\left(X, I_{M}\right) \stackrel{\operatorname{Hom}_{\mathcal{B}}(X, \alpha)}{\longrightarrow} \operatorname{Hom}_{\mathcal{B}}(X, \bar{\Omega} M) \rightarrow \operatorname{Ext}_{\mathcal{B}}^{1}(X, M) \rightarrow 0 .
$$

Moreover, if $I \in \mathcal{I}$, applying $\operatorname{Hom}_{\mathcal{B}}(I,-)$ as $I \in \mathcal{M}$ and $\mathcal{M}$ is cluster tilting, we get an exact sequence

$$
\operatorname{Hom}_{\mathcal{B}}\left(I, I_{M}\right) \stackrel{\operatorname{Hom}_{\mathcal{B}}(I, \alpha)}{\longrightarrow} \operatorname{Hom}_{\mathcal{B}}(I, \bar{\Omega} M) \rightarrow \operatorname{Ext}_{\mathcal{B}}^{1}(I, M)=0
$$

and therefore

$$
\operatorname{Ext}_{\mathcal{B}}^{1}(X, M) \simeq \operatorname{Hom}_{\mathcal{B}}(X, \bar{\Omega} M) / \operatorname{Im}\left(\operatorname{Hom}_{\mathcal{B}}(X, \alpha)\right)=\overline{\operatorname{Hom}}_{\mathcal{B}}(X, \bar{\Omega} M) .
$$

Hence, if $n=2$, we have

$$
\begin{aligned}
\overline{\operatorname{Hom}}_{\mathcal{B}}\left(M, \tau_{1} X\right) & \simeq \operatorname{DExt}_{\mathcal{B}}^{1}(X, M) \simeq \overline{\operatorname{DHom}}_{\mathcal{B}}(X, \bar{\Omega} M) \\
& \simeq \bar{D}_{\overline{H o m}}\left(X, \tau_{1} \tau_{1}^{-1} \bar{\Omega} M\right) \\
& \simeq \operatorname{Ext}_{\mathcal{B}}^{1}\left(\tau_{1}^{-1} \bar{\Omega} M, X\right)
\end{aligned}
$$


which implies that $G\left(\tau_{1} X\right)(M) \simeq \mu(F X)(M)$. As all the isomorphisms used are functorial in $M$ and $X, G \tau_{1} \simeq \mu F$ as functors.

When $n \geq 3$, we get an exact sequence

$$
0=\operatorname{Ext}_{\mathcal{B}}^{i}\left(X, I_{M}\right) \rightarrow \operatorname{Ext}_{\mathcal{B}}^{i}(X, \bar{\Omega} M) \rightarrow \operatorname{Ext}_{\mathcal{B}}^{i+1}(X, M) \rightarrow \operatorname{Ext}_{\mathcal{B}}^{i+1}\left(X, I_{M}\right)=0
$$

for $i \geq 1$. Thus we have $\operatorname{Ext}_{\mathcal{B}}^{i}(X, \bar{\Omega} M) \simeq \operatorname{Ext}_{\mathcal{B}}^{i+1}(X, M)$. Since $X \in$ $\perp_{n-2} \mathcal{M} \subseteq{ }^{\perp_{n-2}} \mathcal{P}$,

$$
\begin{aligned}
\overline{\operatorname{Hom}}_{\mathcal{B}}\left(M, \tau_{n-1} X\right) & \simeq \operatorname{DExt}_{\mathcal{B}}^{n-1}(X, M) \simeq \operatorname{DExt}_{\mathcal{B}}^{n-2}(X, \bar{\Omega} M) \\
& \simeq \operatorname{Ext}_{\mathcal{B}}^{1}\left(\bar{\Omega} M, \tau_{n-1} X\right) \\
& \simeq \operatorname{Ext}_{\mathcal{B}}^{1}\left(\tau_{n-1}^{-1} \bar{\Omega} M, X\right)
\end{aligned}
$$

which also implies $G\left(\tau_{n-1} X\right)(M) \simeq \mu(F X)(M)$. As all the isomorphisms used are functorial, $G \tau_{n-1} \simeq \mu F$ as functors.

\section{EXAMPLES}

In this section, we explain two sources of examples, one coming from Geometry (Cohen-Macauley modules over a singularity) and the other one coming more directly from representation theory (Auslander algebras). These examples have been studied extensively by Iyama (see [14 and [15]) from the point of view of (higher) cluster tilting theory.

Example 4.1. Let $S=\mathbb{C} \llbracket X_{1}, X_{2}, \ldots, X_{d} \rrbracket$ be a formal power series ring in $d$ variables and $G$ be a finite subgroup of $\mathrm{GL}_{d}(\mathbb{C})$ without pseudo-reflections acting on $S$. Let $R=S^{G}$. According to [13, Proposition 13], $R$ is a complete local Cohen-Macaulay ring of Krull-dimension $d$. Then, by [14, Theorem 2.5 ], if $R$ is an isolated singularity, $S$ is a $(d-1)$-cluster tilting object of $\mathrm{CM}(R)$.

It is known by the end of proof of [5. Proposition 2.1] together with the discussion after [3, Proposition 1.1] that $\operatorname{End}_{R}(S) \simeq S * G$ where $S * G$ is the usual skew-group algebra (see also [17]). Thus, in this case, if $d \geq 3$, we deduce from Theorem 3.2 that

$$
\frac{\perp_{d-3} S}{[S]} \simeq \bmod \left(\underline{\operatorname{End}}_{R}(S)\right)^{\mathrm{op}}=\bmod \left(\frac{S * G}{(e)}\right)^{\mathrm{op}}
$$

where the idempotent

$$
e=\frac{\sum_{g \in G} g}{\# G}
$$

corresponds to the trivial representation of $G$.

Remark that, if $R$ is not Gorenstein, then the exact category of CohenMacaulay modules $\operatorname{CM}(R)$ is not Frobenius. Moreover $R$ is Gorenstein if and only if $G \in \mathrm{SL}_{n}(\mathbb{C})$ [22, Theorem 1]. In the Gorenstein case, see also [1] which realizes $\underline{\mathrm{CM}}(R)$ as a generalized cluster category.

For example, if $G=\langle\zeta \mathrm{Id}\rangle$ where $\zeta$ is a primitive $n$-th root of the unity, then

$$
\frac{\perp_{d-3} S}{[S]} \simeq \bmod \mathbb{C} Q / I
$$


where

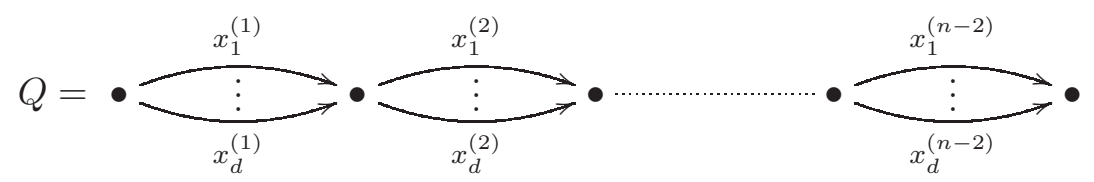

and $I$ is the ideal of $\mathbb{C} Q$ generated by the relations $x_{i}^{(k)} x_{j}^{(k+1)}=x_{j}^{(k)} x_{i}^{(k+1)}$ for $1 \leq i<j \leq d$ and $1 \leq k \leq n-3$ (see [5, 17, 23] for details about the computation).

Recently Herschend, Iyama and Oppermann studied n-representationfinite algebras (see for example [11, 12, 15, 16]), which are finite dimensional algebras with global dimension at most $n$ and has an $n$-cluster tilting module. We can apply our results to all of those algebras. From now on, we describe explicitely a simple example taken from [15].

Example 4.2. Let $\Lambda$ be the Auslander algebra of $k \vec{A}_{3}$. That is $k Q / R$ where $Q$ is the following quiver

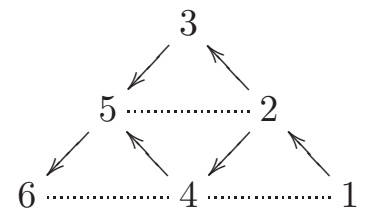

and the ideal of relations $R$ is generated by the mesh relations symbolized by dashed lines. Then, using the method introduced in [15, §1], one can compute a cluster tilting subcategory $\mathcal{M}$ of $\bmod \Lambda$. It is generated by the direct sum of the $\tau_{2}^{i}(D \Lambda)$ for $i \geq 0$ where $\tau_{2}$ is the 2-AR translation.

Let us compute the $\tau_{2}^{i}(D \Lambda)$, each indecomposable module being depicted by its compositions series representation:

$$
\begin{gathered}
D \Lambda=1 \oplus{ }_{2} \oplus{ }_{2}{ }_{3} \oplus{ }_{4}{ }^{2} \oplus 4{ }_{5}^{2}{ }_{3} \oplus{ }_{6}{ }^{3}, \\
\tau_{2}(D \Lambda)=4 \oplus{ }_{5}^{4} \oplus_{6}{ }^{5} \quad \tau_{2}^{2}(D \Lambda)=6 \quad \tau_{2}^{3}(D \Lambda)=0 .
\end{gathered}
$$

The quiver of $\mathcal{M}$ is given in Figure 1

Then we can calculate $\bar{\Omega} \mathcal{M}$ easily since in this case $\bar{\Omega} \mathcal{M}=\overline{\mathcal{M}}^{\perp}=\{X \in$ $\left.\bmod \Lambda \mid \overline{\operatorname{Hom}}_{\Lambda}(\mathcal{M}, X)=0\right\}$. We give a full view of these categories in Figure 2 .

In this example, the quiver of $\bmod \Lambda /[\mathcal{M}]$ is the following.

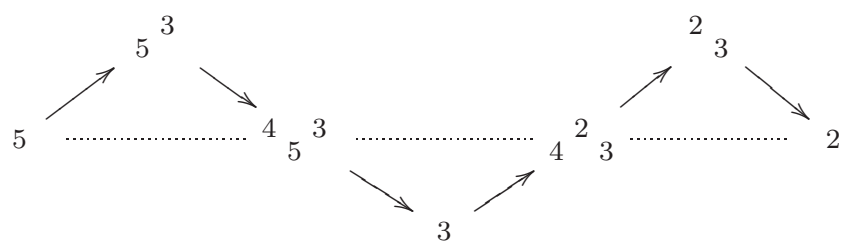

The quiver of $\underline{\mathcal{M}}$ is the following. 


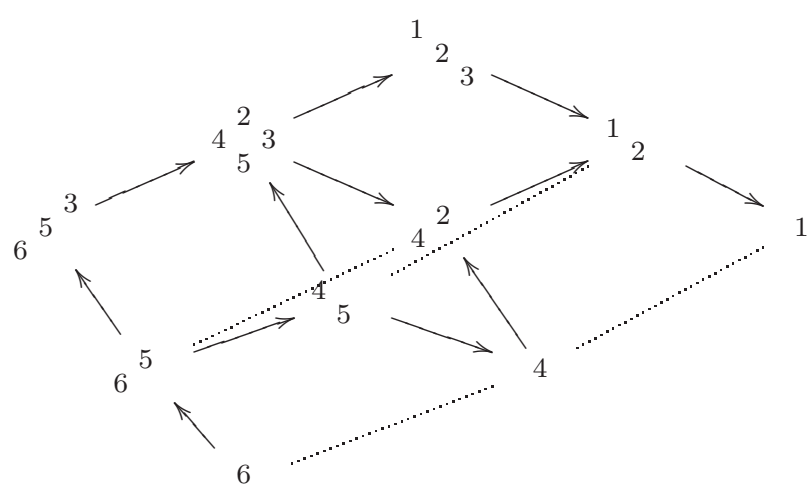

Figure 1. Quiver of $\mathcal{M}$

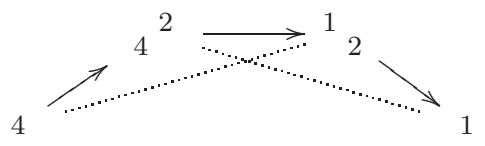

As expected, we obtain that $\bmod \Lambda /[\mathcal{M}] \simeq \bmod \underline{\mathcal{M}}$. One can also calculate and check the equivalence $\bmod \Lambda /\left[\overline{\mathcal{M}}^{\perp}\right] \simeq \bmod \overline{\mathcal{M}}$.

Example 4.3. Keeping previous notation, let

$$
\mathcal{M}^{\prime}=\operatorname{add}\left(6 \oplus{ }_{6}^{5} \oplus{ }_{6} 5^{3} \oplus{ }_{5}^{4} \oplus{ }_{4}{ }_{5}^{2}{ }_{3} \oplus^{1}{ }_{2}{ }_{3} \oplus 4 \oplus{ }_{4}^{2}\right) .
$$

We get $\mathcal{P} \subseteq \mathcal{M}^{\prime} \subseteq \mathcal{M}$. Hence $\mathcal{M}^{\prime}$ is a rigid subcategory of $\bmod \Lambda$ containing all the projective objects. By Theorem 3.2, we get $\mathcal{M}_{L}^{\prime} /\left[\mathcal{M}^{\prime}\right] \simeq \bmod \underline{\mathcal{M}^{\prime}}$. In fact

$$
\mathcal{M}_{L}^{\prime}=\mathcal{M}^{\prime} \oplus \operatorname{add}\left(5 \oplus_{5}{ }^{3} \oplus{ }^{4}{ }_{5}{ }^{3}\right)
$$

and the quiver of $\mathcal{M}_{L}^{\prime} /\left[\mathcal{M}^{\prime}\right]$ is the following:

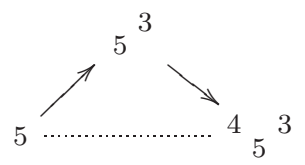

which is isomorphic to the AR quiver of $\underline{\mathcal{M}}^{\prime} \simeq k\left[4 \longrightarrow{ }_{4}^{2}\right]$. 


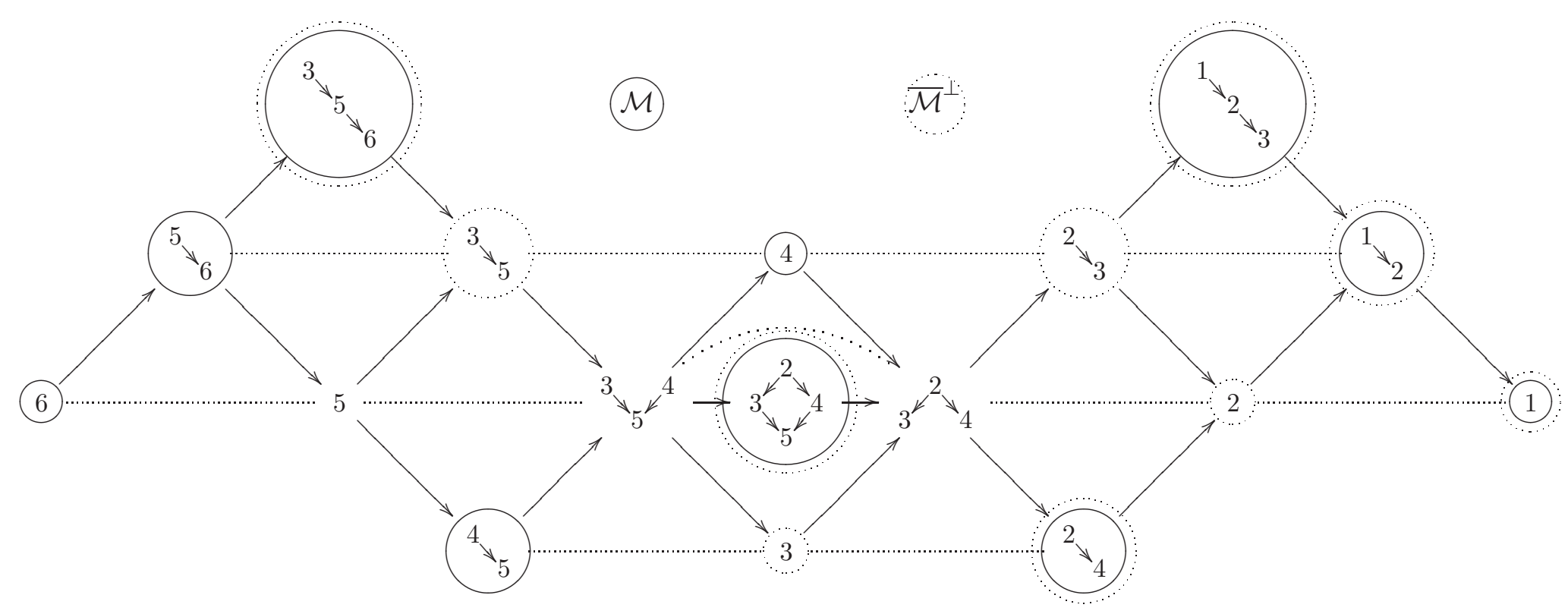

Figure 2. AR-quiver of the Auslander algebra of $k A_{3}$ 


\section{ACKNOWLEDGMENTS}

The authors would like to thank Osamu Iyama for his helpful advises and corrections and the referee for his valuable comments.

\section{REFERENCES}

[1] C. Amiot, O. Iyama, I. Reiten. Stable categories of Cohen-Macaulay modules and cluster categories. arXiv:1104.3658

[2] I. Assem, D. Simson, A. Skowroński. Elements of the representation theory of associative algebras. Vol. 1. Techniques of representation theory. London Mathematical Society Student Texts, 65. Cambridge University Press, Cambridge, 2006. $\mathrm{x}+458 \mathrm{pp}$.

[3] M. Auslander. On the purity of the branch locus. Amer. J. Math. 84 (1962), 116-125.

[4] M. Auslander. Coherent functors. 1966 Proc. Conf. Categorical Algebra (La Jolla, Calif., 1965) pp. 189-231 Springer, New York.

[5] M. Auslander. Rational singularities and almost split sequences. Trans. Amer. Math. Soc. 293 (1986), no. 2, 511-531.

[6] A. B. Buan, R. Marsh, M. Reineke, I. Reiten, G. Todorov. Tilting theory and cluster combinatorics. Adv. Math. 204 (2006), no. 2, 572-618.

[7] A. B. Buan, R. Marsh, I. Reiten. Cluster-tilted algebra. Trans. Amer. Math. Soc. 359 (2007), no. 1, 323-332.

[8] T. Bühler. Exact categories. Expo. Math. 28 (2010), no. 1, 1-69.

[9] C. Geiß, B. Leclerc, J. Schröer. Rigid modules over preprojective algebras. Invent. Math. 165 (2006), no. 3, 589-632.

[10] D. Happel. Triangulated categories in the representation theory of finite-dimensional algebras. London Mathematical Society Lecture Note Series, 119. Cambridge University Press, Cambridge, 1988. $\mathrm{x}+208$ pp.

[11] M. Herschend, O. Iyama. Selfinjective quivers with potential and 2-representationfinite algebras. Compos. Math. 147 (2011), no. 6, 1885-1920.

[12] M. Herschend, O. Iyama. $n$-representation-finite algebras and twisted fractionally Calabi-Yau algebras. Bull. Lond. Math. Soc. 43 (2011), no. 3, 449-466.

[13] M. Hochster, J. A. Eagon. Cohen-Macaulay rings, invariant theory, and the generic perfection of determinantal loci. Amer. J. Math. 93 (1971), 1020-1058.

[14] O. Iyama. Higher-dimensional Auslander-Reiten theory on maximal orthogonal subcategories. Adv. Math. 210 (2007), no. 1, 22-50.

[15] O. Iyama. Cluster tilting for higher Auslander algebra. Adv. Math. 226 (2011), no. $1,1-61$.

[16] O. Iyama, S. Oppermann. $n$-representation-finite algebras and $n$-APR tilting. Trans. Amer. Math. Soc. 363 (2011), no. 12, 6575-6614.

[17] O. Iyama, R. Takahashi. Tilting and cluster tilting for quotient singularities. arXiv:1012.5954, to appear in Math. Ann.

[18] B. Keller. Chain complexes and stable categories. Manuscripta Math. 67 (1990), no. 4, 379-417.

[19] B. Keller, I. Reiten. Cluster-tilted algebras are Gorenstein and stably Calabi-Yau. Adv. Math. 211 (2007), no. 1, 123-151.

[20] S. Koenig, B. Zhu. From triangulated categories to abelian categories: cluster tilting in a general framework. Math. Z. 258 (2008), no. 1, 143-160.

[21] D. Quillen Higher algebraic K-theory. I. Lecture Notes in Math., Vol. 341, Springer, Berlin 1973, pp. 85-147.

[22] K. Watanabe. Certain invariant subrings are Gorenstein. I. Osaka J. Math. 11 (1974), $1-8$.

[23] Y. Yoshino. Cohen-Macaulay modules over Cohen-Macaulay rings. London Mathematical Society Lecture Note Series, 146. Cambridge University Press, Cambridge, 1990. 
QUOTIENTS OF EXACT CATEGORIES BY CLUSTER TILTING SUBCATEGORIES 21 Graduate School of Mathematics, Nagoya University, 464-8602 Nagoya, JAPAN

E-mail address: Laurent.Demonet@normalesup.org

Graduate School of Mathematics, Nagoya University, 464-8602 Nagoya, JAPAN

E-mail address: d11005m@math.nagoya-u.ac.jp 